\title{
Hacia una cultura de paz. Un aporte al debate
}

\section{Towards a Culture of Peace. A Contribution to the Debate}

Jaime Delgado-Rojas

Académico

Posgrado en Derechos Humanos y Derecho Comunitario

Universidad de Costa Rica

Recibido: 2/10/2017 Aceptado: 15/1/2018

\section{Resumen}

En este ensayo, la Cantata de Santa María de Iquique sirve como marco a la reflexión sobre la paz y la alteridad. Plantea que el derecho a la paz es parte integrante de la ética jurídica y política que implica a los "otros" hacia quienes supone, no simplemente tolerancia, sino respeto a las diferencias y convivialidad. El abanico de relaciones con el otro se constituye en enjambre de posibilidades éticas, estimulante y provocador, que nos ubica en el plano de la cultura de paz y en el compromiso de impulsar una educación para la paz y una democracia sustancial.

Palabras clave: Cultura de paz, educación para la paz, América Latina, Derechos Humanos, pensamiento político latinoamericano, alteridad.

\section{Abstract}

In this essay, the Cantata de Santa María de Iquique serves as a framework for reflection on peace and otherness. It argues that the right to peace is an integral part of the legal and political ethic that involves the "others" towards whom it implies not simply tolerance, but respect for differences and conviviality. The range of relationships with the other is a swarm of ethical possibilities, stimulating and provocative, which places us on the level of the culture of peace and commitment to promote an education for peace and a strenghtened democracy.

Keywords: Culture of peace, education for peace, Latin America, Human Rights, Latin American political thought, alterity.

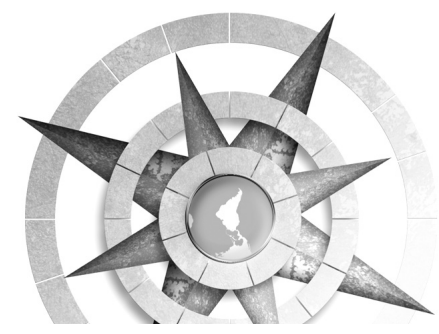


La poesía mueve corazones. Inicio con un canto de protesta contra la injusticia y concluyo con otro, pero de optimismo.

Los Señores de Iquique / tenían miedo; era mucho pedir / ver tanto obrero. El pampino no era / hombre cabal, podía ser ladrón / o asesinar.

Mientras tanto las casas / eran cerradas, miraban solamente / tras las ventanas.

El Comercio cerró / también sus puertas, había que cuidarse / de tanta bestia.

Mejor que los juntaran / en algún sitio, si andaban por las calles / era un peligro.

\section{Santa María de Iquique \\ Cantata popular de Luis Advis divulgada por Quilapayún, Chile, 1971.}

\section{En lo teórico}

Poco nos ayuda el lenguaje dicotómico para expresar la realidad local o mundial, social o económica, política o cultural; sea del socialismo con sus adjetivaciones o del capitalismo, la globalización y la mundialización. Esa realidad múltiple y heterogénea, obliga a echar mano a discursos más comprensivos.

Nos constituimos en sujetos, en entes espirituales, con los intercambios de información, en el diálogo: ese engranaje diverso de comunicaciones que constituye el nosotros, el yo con los otros. En una epistemología dicotómica, a ese otro, como el "pampino", o lo odiamos o lo amamos. La ruptura de esa dicotomía no se logra con la "tolerancia". Tolerar, más bien enuncia la actitud de soportar lo que hace, dice o piensa el otro; acá el lenguaje oculta la arrogancia del yo, el ego emisor del discurso, con su desplante autoritario: tolero, aunque lo que haga, diga o piense el otro no sea lo que hago, pienso o digo. La arrogancia es vertical y excluyente; anula el diálogo que es horizontal y multitemático.

Cuando usamos el lenguaje inclusivo y visibilizamos al otro, nuestro

96 Hacia una cultura de paz. Un aporte al debate Jaime Delgado-Rojas 
discurso se torna horizontal, comunicativo y sugerente. En primer lugar, entre las conductas extremas del rechazo o la identificación, hay otras prácticas posibles: el odio, en el extremo negativo, suele cargar miedos y promover la muerte, como sucede en el norte del continente en colegios, bares y discotecas. De ninguna fobia emerge la paz; tampoco del rechazo, la indiferencia, la invisibilización y, como lo he señalado, la tolerancia.

Como dice el poeta costarricense Jorge Debravo (Nosotros los hombres, 1966): Estamos sin amor, hermano mío, y esto es como estar ciegos en mitad de la tierra.

En lo opuesto, las prácticas positivas posibles hacia el otro, hay respeto, admiración, identificación y amor.

He hecho una ampliación de un esquema usado por el Dr. Virgilio Zapatero, exrector de la Universidad de Alcalá de Henares, no obstante que le da un pedestal positivo a la tolerancia (Zapatero, 2011). Para él, tolerar al otro es aceptarlo en sus prácticas y costumbres, considerándolas válidas: muy particularmente se refiere al inmigrante. El cristianismo clásico, en cambio, es mucho más radical, enseña el amor al prójimo, como fuerza espiritual que sale del yo, como acto de aceptación, desprendimiento (caridad) y entrega. El ego arrogante da paso al sujeto que existe mientras establece el diálogo en el tanto hace reconocimiento del otro.

Boaventura de Sousa Santos hace un planteamiento progresivo de relaciones societales. Habla de sociabilidad y señala cuatro clases: la violencia, expresión y resultado de esquemas socioculturales irreconciliables que, a nuestro entender, se legitima para suprimir, marginar o destruir al otro. En el desplazamiento hacia lo positivo le sigue la coexistencia que, al igual que el concepto de tolerancia, no es más que la aceptación de otro diferente y extraño.

Vendrá la etapa de la conciliación, una sociabilidad que mira al pasado, orientada a sanar las heridas de la confrontación y, por ende, retrospectiva sin saldar sus desequilibrios conceptuales y jurídicos. No garantiza la erradicación de la discriminación, por lo que debe dar paso a la convivialidad, un estadio para mirar y construir el futuro, saldar el pasado, lo que no significa esconderlo, y comprender al otro en su integridad (de Sousa Santos, 2010: 106 y ss.). Lo particular es la progresividad de estas sociabilidades con el proceso de construcción de la paz, desde situaciones negativas a otras promisorias;

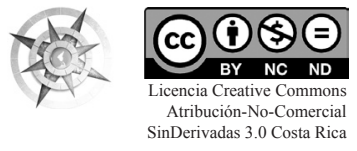


unas con cara hacia el pasado y otras mirando al porvenir.

\section{Diversidad}

Que el otro es diverso es una verdad de Perogrullo. Es una realidad universal, aunque el Dr. Zapatero se refiera al inmigrante en España. En la sociedad costarricense es el nicaragüense, pero hay cubanos, colombianos, africanos y haitianos. También indígenas que no son extranjeros, pues los ngöbes (o guaymíes) son panameños y ticos en cuanto la frontera se les implantó en el medio de su territorio, al igual que en el Caribe centroamericano con los garífunas, miskitos, afrocaribeños, etc.

Ha sido noticia que en la defensa del territorio lenca contra el capitalismo salvaje, fue asesinada la activista Berta Cáceres, indígena ambientalista hondureña.

Un otro en Costa Rica es el negro afrodescendiente; repudiado hasta avanzada la primera mitad del siglo XX cuando se le permitió su paso del $\mathrm{Ca}$ ribe al Valle Central, aunque muchos tengamos ancestros negros comerciados durante la colonia, lo que ha ido siendo rescatado culturalmente.

Es otro el ateo y el agnóstico para los creyentes y en Costa Rica aún vivimos en un Estado confesional estampado en el artículo 75 de la Constitución Política que obliga al juramento ante Dios para cumplir la constitución o confesar la verdad.

Hay repudio al intelectual por temor al pensamiento libre, lo que se expresa en las críticas desde la gran prensa y la derecha militante a las universidades públicas y a los que se dedican -académicos o estudiantesa algunas carreras "peligrosas". La novela 300 (EUNA, 2011) del autor y académico guatemalteco-costarricense, Rafael Cuevas-Molina, retrata las desapariciones durante una de las dictaduras guatemaltecas:

Fue secuestrado por cuatro hombres armados, vestidos de civil, que se desplazaban en dos vehículos, uno de ellos un pickup rojo con vidrios polarizados. La víctima era estudiante de Antropología y catedrático auxiliar de Sociología. A las cinco de la tarde salió del Instituto Nacional de Administración Pública, donde trabajaba, para impartir clases en la Universidad de San Carlos y cuando transitaba por la $5^{\mathrm{a}}$ avenida, entre la 13 y 14 calle de la zona 9 se produjo la detención (pp. 49-50).

Para los defensores del statu quo, el revolucionario es un enemigo y la propaganda agita el miedo, como con los pampinos en la Cantata,

98 Hacia una cultura de paz. Un aporte al debate Jaime Delgado-Rojas 
para que la inacción política sea la defensa del statu quo, sin valorar que en la utopía que mueve la conducta de esa izquierda se reivindica a desposeídos que, en su inacción defienden el statu quo.

En contextos de crisis de legitimidad, es calificado de peligroso el que habla, se reúne o el que participa. La invocación al apoliticismo es el mejor instrumento de dominación de los poderosos: no ir a la marcha, no estar en la huelga, no ir a votar. En esto los medios de comunicación juegan un papel fundamental. El que haya diálogo genera miedos ajenos, pues lo hay intercultural, interétnico, interconfesional y también interjudicial, lo que permite el intercambio de saberes, ilusiones, proyectos, cosmovisiones y de ahí toma de conciencia.

El miedo también construye utopías regresivas y conductas de violación a derechos en las masas: es el populismo de las derechas que moviliza pasiones no solo en Europa y Estados Unidos y que puede opacarnos. También el miedo a la subversión genera violaciones a los derechos fundamentales que no deben olvidarse por "amnistías irrestrictas, absolutas e incondicionales", como lo ha sancionado la Sala de lo Constitucional salvadoreña.
Hay otros que se han lanzado a la conquista de la democracia de la calle, beligerantes por la diversidad de género, con un conjunto de siglas y términos que se tornan extraños en sus significados: LGTTBI, los queer de Norteamérica, los muxes de Oaxaca, perseguidos, ocultados e incomprendidos.

En cuanto a las mujeres su lucha se ha convertido en "la inversión cultural más importante"; según Touraine, y lo cito:

Lo que está en cuestión (...) (es) la afirmación de que lo universal humano no se encarna en una figura, la del Hombre, (...) sino la dualidad del hombre y la mujer que dan forma, a veces de manera diferente, otra de manera idéntica, al mismo proceso de combinación de un ser particular y una racionalidad general, sustancial o instrumental (2000).

Y ese Hombre, con mayúscula, ¿no es sino la expresión del autoritarismo cultural de la sociedad patriarcal, esta prehistoria de la humanidad que persiste en muchas mentes? Continúo con Touraine:

No se trata de una reivindicación particular, de la acción de una minoría, y las feministas ( ) tienen razón en rechazar airadamente la definición de las mujeres como

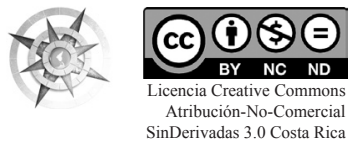


minoría. Puesto que la crítica efectuada por las mujeres tiene un valor general: se trata de destruir la identificación de la cultura o de la modernidad con un actor social particular ( ), que encierra con ello a los otros actores en una condición de inferioridad y dependencia (2000: 40-41).

Y yo pregunto: ¿esa "identificación de la cultura o de la modernidad" no es sino la arrogancia de un yo colectivo, el super ego de que hablaba Freud?

No puedo eludirlos: los indigentes, los pobres o marginados de la ciudad, el "pampino" urbano; esos que viven en la acera, al lado del negocio que los lanza con los cartones a la basura. Muchos son agredidos por los dueños del negocio turístico. He visto un documental que señala que en las urbes europeas se les están poniendo obstáculos metálicos para que no se acuesten a dormir en las bancas de los parques, como si fuese una forma más noble de eliminarlos del paisaje. Hay ciudades de Nuestra América donde los asesinan.

Otros, los desaparecidos por las dictaduras y sus hijos, que en Argentina han ido siendo rescatados de a poquito por las Abuelas de la Plaza de Mayo. Un canto a la vida de estas luchadoras, contra los que les ocultaron y robaron su futuro y un mensaje de esperanza para los que aún siguen sin saber quiénes son sus progenitores. También, las osamentas escondidas en fosas en Guatemala "que hablan en voz baja, nunca mienten y nunca olvidan" (Snow, citado por Jones, 2016).

"Traigo muertes para asustar a todos los que juegan con muertes" (1966), escribía Jorge Debravo. En fin, la paz no se construye enterrando el pasado. Tampoco con invisibilizaciones.

La dicotomía visibilizar e invisibilizar nos abre los ojos. Está invisibilizado el trabajo de la mujer. Están en el clóset los gais y las lesbianas a quienes la sociedad patriarcal los obliga a esconderse de la vergüenza de sus familias. Pero también está el autoocultamiento, cuando la presión del entorno obliga a usar una máscara para esconder algo muy propio. El escritor Sergio Ramírez en su novela La fugitiva dice de la inmigración nicaragüense en Costa Rica:

Los inmigrantes nicaragüenses (...) despiertan miedos y recelos como si en una invasión concertada se prepararan a apoderarse del país, mientras ellos, precavidos, se apresuran a borrar toda huella de su acento y de sus modales confianzudos y a veces agresivos, convencidos de que la mejor manera de protegerse frente a la 
hostilidad es mimetizarse aprendiendo a hablar y a comportarse en la sosegada clave costarricense lo más rápidamente posible, aunque a veces el disfraz no resulte a la medida (2011: 20).

Hay otros ocultamientos: el menos noble (y ¿cuál es más noble?) es la información comandada por los dueños de la prensa y la ideología oficial; otrora la Iglesia, en cualquiera de sus expresiones. Hoy la empresa periodística que se enmascara de defensora del principio de libertad de prensa para ejercer la rentable libertad de empresa de la comunicación (defendida por la SIP), que nos comunica con entusiasmo las delicias de la globalización comercial, como si todo, incluso la intimidad, pudiese circular en calidad de mercancías. Es el otro mundo que nos integra con violencia, mientras nos pulveriza culturalmente, como lo señala Touraine. Porque la información veraz también es un derecho violentado, no hay paz en la desinformación.

El abanico de relaciones con el otro se constituye en enjambre de posibilidades éticas estimulante y provocador. Nos ubica en el plano de la cultura de paz y en el compromiso de impulsar una educación para la paz y una democracia sustancial.

\section{Cultura de paz}

Educar para la paz es una tarea en todo ámbito, pero, en particular, como lo establece la Ley colombiana 1732 del 2014, en la enseñanza básica formal. Respeto, admiración, develación o visibilización son expresiones de la cultura de paz. Cultura no es la pasión por la erudición, el intelectualismo, ni la admiración atónita del mundo europeo, como se usó durante el periodo de construcción de nuestros Estados nacionales; sino, como se indicó en la Conferencia Mundial de Políticas Culturales de la UNESCO (México, 1982) es: "el conjunto de los rasgos distintivos, espirituales y materiales, intelectuales y afectivos que caracterizan a una sociedad o un grupo social". Comprende las artes y las letras, los modos de vida, los derechos fundamentales del ser humano, los sistemas de valores, las tradiciones y las creencias. Está imbricada en los contenidos de la identidad cultural que representa y es, vuelvo a citar, "un conjunto de valores único e irreemplazable, ya que las tradiciones y formas de expresión de cada pueblo constituyen su manera más lograda de estar presente en el mundo". (citado por Delgado, 2011: 24).

Desde esta definición, la cultura de paz confronta la cultura de guerra y 
la clásica educación para la guerra estampada en el proverbio latino de Vegecio Renato: si vis pacem para bellum o qui desiderat pacem, praeparet bellum (quien desee la paz que prepare la guerra). Cuando impulsó la Universidad para la Paz en 1980, el recordado expresidente costarricense Rodrigo Carazo asentó su visión de que si se quiere la paz hay que educar para la paz. El lema es: si vis pacem para pacem.

La cultura de paz nada tiene que ver con la integración social o con asimilaciones funcionales. Muchas barbaridades se han cometido con los intentos funcionalistas de promover la homogeneización a la fuerza. No lo lograron los conquistadores imponiendo el culto del cristianismo; más bien provocó genocidio. Tampoco con la paz de cementerios promovida por los Estados de seguridad nacional que actuaban como Leviatanes pacificadores de estados de naturaleza hobbesianos, con violación de los derechos fundamentales. Por ello la cultura de paz no se construye con amnistías irrestrictas, absolutas e incondicionales, como lo ha señalado la Sala de lo Constitucional salvadoreña:

Si bien la Constitución, el Derecho Internacional Humanitario, el Derecho Internacional de los Derechos Humanos y la jurisprudencia internacional en materia de derechos humanos, permiten la adopción de amnistías, incluso a la cesación de las hostilidades militares tras la finalización de conflictos armados (...), ello no implica que el Órgano Legislativo esté habilitado para decretar amnistías irrestrictas, absolutas e incondicionales, desconociendo las obligaciones constitucionales e internacionales que tienen los Estados en lo relativo a la protección de los derechos fundamentales, de investigar, identificar a los responsables materiales e intelectuales, y sancionarlos conforme a su derecho interno; desconociendo, además, el deber de reparar integralmente a las víctimas de crímenes de lesa humanidad y crímenes de guerra constitutivos de graves violaciones al Derecho Internacional Humanitario (pág. 2).

Vivimos en el multiculturalismo, en una multiplicidad de visiones del mundo y actores.

El lado oculto de ese multiculturalismo, dice Touraine, es el riesgo de encierro de cada cultura en una experiencia particular incomunicable. Semejante fragmentación cultural nos conduciría a un mundo de sectas y al rechazo de toda norma social (2000: 41). Sobre todo, cuando esa norma social es la exigencia de

102 Hacia una cultura de paz. Un aporte al debate Jaime Delgado-Rojas
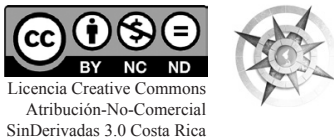
ocultamiento, como con los "pampinos" de la Cantata, el acento en su lenguaje de los nicaragüenses, o los géneros diversos.

La inclusividad es la aceptación respetuosa del otro en sus diferencias. Para ello se requiere forjar la cultura de paz que permita acercarnos al vecino en el barrio, en la provincia o Estado, en la Nación y en la Región. ¡Cuánto nos inventamos para no aceptar al nica, al colombiano, al cubano, en Costa Rica! y ¡cuántas justificaciones se hacen para no dejar pasar hacia el Norte a migrantes de este y el otro continente!, mientras el Norte (el capitalismo salvaje) los atrae con sus cantos de sirena. Y ¡cuántas realidades nos acercan como hermanos! Creo que aún no ha sido contabilizada la riqueza cultural y económica que les debemos a los inmigrantes: intelectuales del Sur que huyeron de dictaduras y se incorporaron a nuestras universidades a coadyuvar en la formación profesional, la investigación y el forjamiento de un sentido de identidad latinoamericana; los médicos de Cuba en nuestras clínicas y los trabajadores y las trabajadoras nicaragüenses en el campo, en la vigilancia y en la cocina.

\section{Integración y paz}

Me referiré, en términos muy generales, a los procesos de integración regional en este continente. Frente a la agresión real o posible del entorno, la convocatoria a la Asamblea General Americana, celebrada en Panamá en 1826 para la discusión, aprobación y firma del "Tratado de Unión, Liga y Confederación Perpetua" (22/6 al 5/7 de 1826), fue un avance en la construcción de la paz, como autodeterminación. El acuerdo tenía connotación defensivista y sentido westfaliano, frente a la amenaza de la Santa Alianza, con expresiones muy sólidas: la Doctrina Monroe de 1823 y el Imperio Brasileño, de los Braganza desde 1807, único experimento monárquico exitoso en el continente.

La Santa Alianza reafirmaba el estado westfaliano constituido al interior de las fronteras hasta donde llega el ejército y en la nación forjada por juristas, diplomáticos y militares, según el primer estilo de construcción de los Estados nacionales definidos por Habermas.

En el siglo XX la cultura de paz en América Latina tendrá el doble juego de amistad y confianza con los vecinos del Sur, pero de posiciones ambiguas o sometimiento a la potencia 
del Norte. En el panamericanismo, desde el Congreso de Washington de 1891-93, se evidenciará esa tensión constantemente, cuyo encuentro inicial fue analizado por José Martí en Nuestra América (1982: 35 y ss.).

Para nuestro objetivo son valiosas las definiciones que se hacen el $26 \mathrm{de}$ diciembre de 1933 en Montevideo, Uruguay, en la Convención sobre la Enseñanza de la Historia. Se recomendaba, entre otras, la eliminación de "los paralelos enojosos entre los personajes históricos nacionales y extranjeros, y los comentarios y conceptos ofensivos y deprimentes para otros países".

En coherencia enseñaba no juzgar "con odio" o falsear "los hechos en el relato de guerras o batallas cuyo resultado haya sido adverso"; al contrario, pedía que se destacara lo que "contribuya constructivamente a la inteligencia y cooperación de los países americanos" (citado por Delgado, 2011: 40 y ss.).

Desde la década de 1950, y acogiendo la herencia bolivariana del periodo emancipatorio, se generaron encuentros e instituciones regionales valiosos y es común encontrar alusiones a la paz, construcciones de zonas de paz y referencias a una cultura de paz, en instrumentos de carácter internacional o regional. Se construyen zonas de paz en las que los Estados reafirman "su compromiso de no recurrir al empleo de la fuerza para resolver los conflictos que puedan existir" entre ellos y en las que las potencias externas "se comprometen a no servirse de esa zona para sus objetivos bélicos o armamentistas", así lo enseña el uruguayo Héctor Gros Espiell (citado por Delgado Rojas, 2011: 91).

La integración centroamericana, por ejemplo, se redefine a fines del siglo $\mathrm{XX}$ a partir de las décadas de guerra subregional, heredando la tradición unionista del siglo XIX. Hay contenidos de cultura de paz en los acuerdos de Esquipulas de 1986 y 1987, en el Tratado de Tegucigalpa de 1991 que crea el SICA y en el Tratado Marco de Seguridad Democrática de 1995, los que distan mucho de la visión bélica que había en las Cartas de San Salvador de 1951 y 1962 constitutivas de la ODECA.

Mientras en el resto del continente hay acuerdos que involucran la dimensión territorial continental (Río, 1947), otro, la latinoamericana (Tlatelolco, 1969), un acuerdo integra a todo el Caribe (1979), otro se reduce al ámbito ribereño del Atlántico Sur (1986), hay en el Mercosur (1998) y en la Comunidad Andina 
(1989, 2002 y 2004), pero también está el acuerdo de toda América del Sur (2002). En estos instrumentos de derecho internacional latinoamericano, la paz se concibe como resultado deseable de un conflicto existente o potencial en el que participan, al menos dos Estados.

Pero esa paz hacia afuera del Estado tampoco puede construirse sin una cultura de paz hacia adentro de la Nación, como se recomendaba en la Convención de Montevideo de 1933. El tema es nacional y regional mucho más ahora que las fronteras han sido desdibujadas por múltiples procesos sociales, culturales y circunstancias políticas.

Con la creación de la Unión de $\mathrm{Na}$ ciones de Sudamérica, esta idea de una zona subcontinental de paz queda formulada en el acuerdo constitutivo de la UNASUR, en el 2008. Las alusiones son a contenidos de la cultura de paz más amplios:

(...) irrestricto respeto a la soberanía, integridad e inviolabilidad territorial de los Estados; autodeterminación de los pueblos; solidaridad; cooperación; paz; democracia; participación ciudadana y pluralismo; derechos humanos universales, indivisibles e interdependientes; reducción de las asimetrías y armonía con la naturaleza para un desarrollo sostenible.

En el 2012, en Lima:

La promoción en la región de una cultura de paz basada, entre otros, en los propósitos del Tratado Constitutivo de UNASUR y en los principios de la Declaración y Programa de Acción sobre Cultura de Paz de las Naciones Unidas.

En la Cumbre de la CELAC celebrada en La Habana en el 2014, los 34 mandatarios suscribieron una Declaración mediante la que definen esta región como Zona de Paz y donde reiteran los acuerdos y compromisos subregionales anteriores sobre desarme nuclear, paz y cooperación, solución pacífica de controversias, etc. Como cierre, la promoción en la región de una cultura de paz basada en los principios de la Declaración sobre Cultura de Paz de las Naciones Unidas.

Las referencias en UNASUR y CELAC remiten a la resolución de NNUU que dice que la cultura de paz

(...) consiste en una serie de valores, actitudes y comportamientos que rechazan la violencia y previenen los conflictos tratando de atacar sus causas para solucionar los problemas mediante el diálogo y la negociación entre las personas, las naciones, teniendo 
en cuenta un punto muy importante que son los derechos humanos, pero así mismo respetándolos y teniéndolos en cuenta en esos tratados (Resolución 6 de octubre de 1999, 53 periodo de sesiones, NNUU, Acta 53/24353/243).

"La paz, enseñaba Gros Espiell, es una aspiración universal de entrañable raíz humana (...) fundada en una idea común a todos los miembros de la especie humana" (2005: 519): es un derecho humano, aunque no haya un instrumento de derecho internacional que lo legitime. Al igual que en Colombia, se legisló a favor de la cultura de paz en el 2014, en correspondencia con el artículo 22 constitucional, en Costa Rica se aprobó, en el mismo año, una ley que legitima el derecho a la paz, en correspondencia con su artículo 12 constitucional y la Declaración de Neutralidad de 1983:

La paz es un derecho humano fundamental (...) El Estado incluirá (...) en sus programas de educación, principalmente preescolar, primaria y secundaria, contenidos curriculares que propugnen y cimienten la cultura de paz (...) (Proclamación de la Paz como Derecho Humano y de Costa Rica como país neutral. Asamblea Legislativa, Comisión Plena Tercera, 20/11/2014).
Párrafo muy similar a la ley colombiana citada y ambas firmadas el mismo año de la declaración de la CELAC. El derecho a la paz es, entonces, parte integrante de la ética jurídica y política; en tanto solidaridad, justicia y fraternidad implica a los "otros": supone no simplemente tolerancia, sino respeto a las diferencias y convivialidad. Es un compromiso estatal pero también regional.

\section{Hacia qué se lucha}

No es confrontarnos entre el nosotros, sino contra el estado de cosas que nos ha impuesto la globalización y que pretende convertirnos en enemigos. Es contra la exclusión provocada por el capitalismo salvaje que pulveriza la cultura y las identidades. La paz tiene que ver con el esfuerzo ético del encuentro, con el diálogo horizontal y multitemático, con sus diversos adjetivos, según los campos en que se realiza. El que se construye en la convivialidad, el respeto y el amor como acción desprendida e incondicionada. Como lo expresara, hace más de dos siglos, Kant: "obra de tal modo que uses la humanidad, tanto en tu persona como en la de cualquier otro, siempre como un fin y nunca sólo como un medio".

Así el trato a los otros es asumirlos como dueños de sí mismos, con sus 
proyectos vitales, ilusiones, sueños y utopías; con lo que para nosotros pueda ser erróneo, incomprensible e incluso risible, como lo anota Zapatero. Pero también, con lo que pueda sumarse a lo nuestro, en nuestro proyecto y porvenir. Es la convivencia en la utopía común.

No puede haber paz en el trato discriminatorio y ello implica no arrogarnos sentidos de superioridad, autoritarismo y poder y, por ende, obliga a limitarnos a nosotros mismos, a no manipular a los otros, ni convertirlos en simples instrumentos de nuestros intereses: respetar a mi prójimo es, nos enseñaba Kant, no degradar al otro convirtiéndole en medio para mis fines. El respeto es un deber incondicionado.

La tarea es múltiple: familiar, local, de políticas públicas en el espacio del Estado-nación y trasciende al ámbito regional. Los otros, que están aquí y han llegado por diversas razones, han evidenciado en su carne propia, cómo sus sueños de futuro se les frustran en la intolerancia o la invisibilidad: para los que nos sentimos mayoría, esos otros son los del campo o los de afuera, o los que combaten la injusticia y el statu quo, los que ya no se ocultan, ni en el clóset, ni en la mimetización del lenguaje o de sus credos. Con Kant,

una violación del derecho, cometida en un sitio, repercute en todos los demás (...) la idea de un derecho de ciudadanía mundial no es una fantasía jurídica, sino un complemento necesario del código no escrito del derecho político y de gentes, que de ese modo se eleva a la categoría de derecho público de la Humanidad y favorece la paz perpetua, (...) (1795).

Concluyo con nuestro poeta Jorge Debravo: 
Pero la paz, hermanos, compañeros, se acerca.

Viene sobre los montes en hombros labradores, viene sobre los trenes en hombros maquinistas viene sobre los mares en hombros pescadores. Grandes hordas de fieras quieren sembrar la guerra, echan dientes de hidra en todos los terrenos, pintan caras amargas en las ventanas nuevas... Sin embargo, la paz, compañeros, se acerca. Yo la oigo venir sobre las noches. Viene por los montes, las casas, las fábricas, las minas, los terrenos sembrados de frutos y de flores.

La Paz. En Nosotros los hombres. Poemas de Jorge Debravo (1938-1967)

\section{Referencias bibliográficas}

Asamblea Legislativa $(2014,11 / 20)$. Proclamación de la Paz como Derecho Humano y de Costa Rica como país neutral. Comisión Plena Tercera: Costa Rica.

Cuevas Molina, R. (2011). 300. EUNA: Costa Rica.

Debravo, J. (1966). Nosotros los hombres. Editorial Costa Rica: Costa Rica.

Delgado Rojas, J. (2009). Construcciones supranacionales e integración regional latinoamericana. EUCR: Costa Rica.

Gross-Espiell, H. (2005). El derecho humano a la paz. Anuario de Derecho Constitucional Latinoamericano. Konrad Adenauer Stiftung: Uruguay.
Kant, I. (1795). La paz perpetua. Tecnos: España.

Naciones Unidas (6 de octubre, 1999). Resolución 6 de octubre de 1999. Declaración sobre una cultura de Paz. 53 periodo de sesiones. Acta 53/24353/243

Ramírez, S. (2011). La fugitiva. Alfaguara: España.

Touraine, A. (2000). ¿Podremos vivir juntos? Iguales pero diferentes. FCE: México.

UNASUR (2009). Tratado constitutivo de las naciones suramericanas. Consultado de: Cancillería de Brasil http://www. mre.gov.br/portugues/imprensa/nota detalhe3.asp?ID_RELEASE $=5466$

108 Hacia una cultura de paz. Un aporte al debate Jaime Delgado-Rojas 